\title{
On Measuring the Perceived Onsets of Spontaneous Actions
}

\author{
Hakwan C. Lau, ${ }^{1,2,3}$ Robert D. Rogers, ${ }^{4}$ and Richard E. Passingham ${ }^{1,2}$ \\ ${ }^{1}$ Wellcome Department of Imaging Neuroscience, University College London, London WC1N 3BG, United Kingdom, ${ }^{2}$ Department of Experimental \\ Psychology, University of Oxford, ${ }^{3}$ Oxford Centre for Functional Magnetic Resonance Imaging of the Brain, and ${ }^{4}$ Department of Psychiatry and Oxford \\ Centre for Clinical Magnetic Resonance Research, University of Oxford, Oxford OX3 9DU, United Kingdom
}

We investigated the neural mechanisms underlying the timing procedure that was devised by Libet et al. (1983) to measure the onset of conscious motor intentions in spontaneous actions. We previously showed that, when participants were required to estimate the onset of their intentions using this procedure, the activity in the presupplementary motor area (pre-SMA) was enhanced. Here, we show that when participants were required to estimate the onset of their motor executions (instead of their intentions), the activity in the cingulate motor area was enhanced. Across participants, the degree of this neural enhancement was correlated with the degree of perceptual bias: the higher the degree of enhancement, the earlier the perception. Analysis of data from a previous experiment suggests that the same principle holds true for the relationship between the perceived onset of intentions and the activity in the pre-SMA. We therefore argue that the timing method of Libet et al. (1983) is problematic, because the measuring process affects the neural representations of action and thus also the perceived onsets that the method is designed to measure.

Key words: attention; motor intention; motor; movement; motion; motor activity; fMRI; motor control

\section{Introduction}

Libet et al. (1983) famously used a cross-modal timing method to study the chronometry of volition. In these studies, the onset of intention or movement was estimated in terms of the perceived position of a dot revolving quickly around a clock face, this being reported by the participants after the execution of a self-paced action. It was found that the perceived onset of the intention was $\sim 200 \mathrm{~ms}$ earlier than actual movement execution. Interestingly, the perceived onset of movement was also judged to be earlier than the actual movement onset by $\sim 50 \mathrm{~ms}$.

The method of time estimation used in the study by Libet et al. (1983) has a long and controversial history (Boring, 1950; Spence et al., 2001). Critics have raised various potential temporal biases that could be induced by the method (Libet, 1985; Gomes, 1998; Joordens et al., 2002; Klein, 2002; Trevena and Miller, 2002). However, there has been a lack of empirical investigations of the actual contribution of these biases. Because some of these potential biases operate at opposite directions, it has even been speculated that they might cancel each other out (Klein, 2002). However, this could not explain why the perceived onset of movement was judged to be earlier than the actual movement.

The present study investigates whether this negative bias in perceived movement onset reported by Libet et al. (1983) was attributable to some idiosyncratic features of their method of measurement. In a previous functional magnetic resonance imaging (fMRI) study (Lau et al., 2004), we found that, when participants used the method of Libet et al. (1983) to measure the onset of intention, there was enhanced activity in the medial

Received July 25, 2005; revised April 30, 2006; accepted May 23, 2006.

This work was supported by a Rhodes scholarship (H.C.L.) and the Wellcome Trust (R.E.P.).

Correspondence should be addressed to Hakwan C. Lau, Functional Imaging Laboratory, 12 Queen Square, London WC1N 3BG, UK. E-mail: h.lau@fil.ion.ucl.ac.uk.

D01:10.1523/JNEUROSCI.1138-06.2006

Copyright $\odot 2006$ Society for Neuroscience $\quad$ 0270-6474/06/267265-07\$15.00/0 frontal cortex. An EEG study (Sirigu et al., 2004) revealed a similar result, in that the readiness potential (Deecke et al., 1969; Deecke, 1987) preceding movement showed both enhanced intensity and an earlier onset. We hypothesized that a similar phenomenon of enhancement of neural activity would probably be found for the judgment of the onset of movement, and that such enhanced activity may be associated with the error in the onset judgment in a systematic manner. In particular, if this enhancement of activity reflects more effective information processing and improved the accuracy of temporal judgments as one might expect, it should be associated with a decreased level of negative bias in the perceived movement onset. This would predict that, across subjects, the degree of enhancement of neural activity would show a positive correlation with the perceived movement onset. On the other hand, if this enhancement of activity actually contributes to the negative bias, one would expect a negative correlation. The former hypothesis is motivated by the general finding that task-related enhancement of neural activity in general heightens performance. The latter hypothesis is supported by the recent EEG study by Sirigu et al. (2004) that performing the Libet timing task shifts the onset of the bereitschaftspotential to be earlier. Based on the results in the present study, we also analyzed the data of our previous fMRI study (Lau et al., 2004) to test whether the same principle would hold true for the judgment of the onset of intentions.

\section{Materials and Methods}

Psychological tasks. Fifteen healthy right-handed participants participated in the experiment, which involved four experimental conditions. The "action timing" condition was based on the experimental procedures in Libet et al. (1983). In every trial, participants watched a red dot revolving around an unnumbered but calibrated clock face (diameter $\approx$ $3^{\circ}$ ) at a speed of $2560 \mathrm{~ms}$ per cycle. They were required to fixate their gaze at a cross presented in the middle of the clock face, and press a button with their left index finger at a random time after the dot has finished the 
Table 1. Design of experiment

\begin{tabular}{lll}
\hline & Modality & \\
\hline Timing & Action timing & Auditory timing \\
& Action nontiming & Auditory nontiming \\
\hline
\end{tabular}

The experiment employs a $2 \times 2$ factorial design with modality and timing as the experimental factors. The inclusion of the auditory control conditions allowed us to look for modulation that was driven by the need to perform time estimation using the cross-modal method, which were specific to actions but were not simply a general consequence of the timing process; this is picked out by testing the interaction of the two experimental factors.

first revolution. They were required to act as spontaneously as they could and in particular to avoid first choosing a position of the dot and then using this to trigger the movement. After the button was pressed, the dot disappeared after a variable period of $1280-2560 \mathrm{~ms}$. A random variable delay was used so that subjects could not use the point where the dot disappeared to infer when they had pressed the button. After an additional $4-10 \mathrm{~s}$ variable delay period, the red dot appeared again at the middle of the clock. The participants used a game-pad with their right thumb to control the dot on the screen as a cursor. They were required to move the dot to where it was on the clock face when they pressed the button. After the cursor stayed still for $1 \mathrm{~s}$, it disappeared, and the position of the dot was recorded by the computer, and the difference in the recorded position and the position during the onset of the button press was translated into milliseconds by using the fact that $1^{\circ}=7.1 \mathrm{~ms}$.

In the "action nontiming" condition, the dot disappeared after revolving for one cycle. The participants were required, as in the action timing condition, to make a spontaneous button press. When the button was pressed, the red dot reappeared briefly (duration, $200 \mathrm{~ms}$ ) at a random location around the clock face and the participants were required to remember this location, which they have to report after a $4-10 \mathrm{~s}$ variable delay period using the same method as in the action timing condition.

As in the original studies of Libet et al. (1983), stimuli of a different modality were included in the experiment. The "auditory timing" condition was similar to the action timing condition except that no spontaneous action was required. Instead, an auditory tone of $1 \mathrm{~s}$ duration was presented at $95 \mathrm{~dB}$ through headphones to the participants at a random time (500-5120 ms after the first revolution of the clock). However, the participants were wearing earplugs so that, at this volume, the tones were clearly audible but not unpleasantly loud. After the presentation of the tone, the moving disappeared after a variable period of 1280-2560 ms. The participants were required to estimate the onset of the tone instead of the onset of the button press.

The "auditory nontiming" condition was similar to the auditory timing condition, in the same way that no spontaneous action is required but an auditory tone was presented at a random time (500-5120 ms) after the dot disappeared on completing the first revolution. The dot reappeared in a random location when the tone was presented. The participants were required to remember and later report this location.

These tasks were grouped into blocks of 12 trials of the same condition, and between each trial there was a $4-10 \mathrm{~s}$ variable delay. There were three blocks of each condition in total, and they were presented in a random order. After every four blocks, there was a short break of $30 \mathrm{~s}$. The entire experiment lasted for 46.3-52.2 $\mathrm{min}$ (mean, $49.5 \mathrm{~min}$ ).

Experimental design. The four task conditions constitute a $2 \times 2$ factorial design with "modality" and "timing" as experimental factors (Table 1). The contrast for the timing factor compares the timing conditions against the nontiming conditions and was set up to pick out activities in the brain that are relevant for performing the cross-modal timing task concerned here. The contrast for the modality factor compares the action conditions against the auditory conditions and was set up to pick out activities that specific to the either action or sound. The inclusion of the auditory control conditions allowed us to look for modulation that was driven by the need to perform time estimation using the cross-modal method, which were specific to actions but were not simply a general consequence of the timing process; this is picked out by testing the "interaction" of the two experimental factors.

Data collection. All participants gave informed consent and received MRI safety screening before the experiment. The fMRI data were acquired in a 3 T whole-body MRI scanner (Siemens, Erlangen, Germany).
A quadrature birdcage head coil was used. Head movements were restrained with soft pads. Echo planar imaging (EPI) images were acquired continuously with a repetition time of $3 \mathrm{~s}$ and at an image resolution of $3 \times 4 \times 5 \mathrm{~mm}$. The echo time and effective flip angle were $30 \mathrm{~ms}$ and $90^{\circ}$, respectively. Slices were acquired in axial orientation parallel to the anterior-posterior commissural line, and covered the entire brain volume. After the participants finished the psychological tasks in the scanner, a B0 field map was acquired. A high resolution $(1 \times 1 \times 1.5 \mathrm{~mm})$ T1 structural image was also acquired for participants who have not participated in previous studies in the center before.

Data analysis. The fMRI data were analyzed with statistical parametric mapping, using the SPM2 software (Wellcome Department of Imaging Neuroscience, London, UK). The first four scans of all EPI series were excluded from the analysis to minimize T1 relaxation artifacts. A mean image for all scan volumes was created, to which individual volumes were spatially realigned by rigid body transformation. Unwarping was performed during realignment to correct for dynamic motion-distortion interaction artifacts (Andersson et al., 2001). Static geometric distortions were then corrected with the B0 field map obtained during the experimental session (Jezzard and Balaban, 1995), using Field-map Undistortion Toolbox (R. Cusack, Medical Research Council, Cognition and Brain Sciences Unit, Cambridge, UK). The high-resolution structural image was coregistered with the mean image of the EPI series, and was segmented into gray- and white-matter images. The gray-matter image was normalized to the a priori gray-matter template produced at the Montreal Neurological Institute (Montreal, Quebec, Canada). The normalization parameters were then applied to the EPI images to ensure an anatomically informed normalization. The resulting images were subsampled into a resolution of $2 \times 2 \times 2 \mathrm{~mm}$. A Gaussian filter of $10 \mathrm{~mm}$ full width at half-maximum was then applied to smooth the data spatially, to take into account the anatomical variability between participants, and to satisfy the assumptions of Gaussian random field theory, which was used for the correction of multiple comparisons in the analysis (Worsley et al., 1996).

We expected that the total brain activations for the timing conditions might be much higher than those of the nontiming conditions, because the former are more demanding tasks. Therefore, to make sure that the within-area comparisons between these conditions were valid, global normalization was not performed. The time series data at each voxel were processed using a high-pass filter with a cutoff of $128 \mathrm{~s}$ to remove lowfrequency drifts. Short-term serial correlations in the data were modeled by an auto-regressive process with white noise (Worsley et al., 2002).

The subject-level statistical analyses were performed using the general linear model. The events of interest were the onsets of the button presses and auditory tones in the four experimental conditions, respectively. They were all modeled as events of zero duration. The periods during which participants made their responses using the game-pad were also modeled as an effect of no interest, because activity related to these periods was likely to be associated with the motor areas. The response times were used as the durations for each trial.

Previous results (Lau et al., 2004) suggest that, in this paradigm, the hemodynamic responses reach their maxima well before 5-6 s. Therefore, to avoid making strong assumptions about the heomodynamic responses, the vectors containing the events modeled were convolved with a Fourier set of two sine, two cosine, and one envelope function of a time window of $16 \mathrm{~s}$. This length of the time window was chosen because the hemodynamic activity typically falls back to a near baseline level by $16 \mathrm{~s}$ after stimulation, to maximize the efficiency to precisely model the hemodynamic response we adopted a window length of this magnitude; a wider window would require more regressors to be included to produce a precise fit. Together with a constant term, the regression matrix thus contained 26 columns, and each event was characterized by five parameter estimates. To assess the results of individual subjects, $f$ contrasts were created to test the effects of the main experimental factors (modality and timing) and their interactions jointly determined by the five parameter estimates, in a manner that has been documented in more detail in previous studies using Fourier sets (Ramnani and Miall, 2003). The simple effect of action timing versus action nontiming was also assessed using an 
Table 2. Summary of fMRI results

\begin{tabular}{lllrl}
\hline Effect & Region & Coordinates & Voxels & Maximum Z \\
\hline Timing & Frontal eye field (R) & $24,6,58$ & 562 & 5.83 \\
& Superior parietal cortex (L) & $-22,-66,56$ & 407 & 5.40 \\
& Posterior intraparietal cortex (R) & $24,-60,60$ & 639 & 5.34 \\
& Anterior intraparietal cortex (R) & $54,-24,50$ & 11 & 4.75 \\
& MT/V5 (R) & $44,-64,-2$ & 133 & 5.24 \\
Modality & SMA/cingulate & & & 5.96 \\
& Primary motor cortex (R) & $-6,0,52$ & 1668 & 7.57 \\
& Superior temporal sulcus (R) & $70,-24,52$ & 2513 & 5.42 \\
Timing $\times$ modality & Supramarginal gyrus (L) & $-60,-30,34$ & 96 & 5.07 \\
\hline
\end{tabular}

The results were thresholded at $p<0.05$, corrected for multiple comparisons across the entire brain volume, except for the cingulate motor area, which was small volume corrected based on the result of SMA/cingulate in the modality contrast. L, Left; $R$, right.

$f$ contrast, which was used to identify the voxels for the correlation analysis (see below).

To perform the group-level random effects analysis, $t$ contrasts for each of the five parameter estimates were created for each subject to assess the effects of the main experimental factors and their interactions, which means $15 t$ contrast images were created for each subject. Three separate ANOVA models were set up to assess the effects of the two main experimental factors and their interactions, respectively. For each model, each of the five sets of contrast images corresponding to a particular parameter estimate for all subjects was entered into the model as a condition. Nonsphericity correction was performed with correlated repeated measures considered and by taking subjects as replications. An $f$ contrast that takes the form of a $5 \times 5$ identify matrix was set up to assess the results of each model, which means the null hypothesis was that the contrasts of parameter estimates for all five components of the Fourier set were all zero.

All results were assessed with an extent threshold of 10 voxels. All positive results reported were thresholded at a height threshold of $p<$ 0.05 , with multiple comparisons corrected either across the entire brain volume or within a small volume defined independently from the contrast of interest. Figures 1-4 were generated based on these thresholds, overlaying the results onto the canonical single subject's brain (Montreal Neurological Institute) provided in the SPM software. The presented time courses of hemodynamic responses are the averages of the fitted responses, and the error bars represent SEs across subjects.

Correlation analysis was performed to test the relationship between the perceived onset of the button presses and the degree of modulation of neural activity from action nontiming to action timing in the cingulate motor region [cingulate motor area (CMA)]. To take into account the individual anatomical variability, the voxel at which the modulation was at its maximum for a particular individual was selected for the analysis. This was done by extracting the $p$ values and fitted responses from the local maximum (for the $f$ contrast statistics of action timing vs action nontiming) nearest to the peak coordinate from the CMA $(-10,-4,48)$ from the interaction analysis. The individual variability of the peak location varied: the maximum ranges for the $x, y$, and $z$ values were 2 to -24 , 4 to -18 , and $60-42$, respectively; the SDs were 7,7 , and $6 \mathrm{~mm}$, respectively. The modulation factor for each individual subject was calculated as the difference between the maxima of the fitted response for action timing and action nontiming, divided by the maximum of the fitted response for action nontiming.

A similar analysis was performed for the data from a previously published experiment (Lau et al., 2004), in which we investigated the neural mechanism underlying the estimation of the onset of intention. The study involved two experimental conditions: "intention" and "movement." Participants estimated, using the same cross-modal procedure, the onset of the intention or the onset of motor execution in the relevant conditions. Data for each individual subject were extracted from the voxel at which the statistical score for the modulation effect (as assessed by a $t$ contrast of the estimates for the canonical HRF in the two experimental conditions) was at the nearest local maximum from the group result at the presupplementary motor area (pre-SMA) $(2,4,54)$. The modulation factor was also calculated based on the maxima of the fitted responses in the way explained above. Because the experiment involved no conditions in which there was no timing process involved, the movement condition was used as the baseline.

\section{Results}

\section{Behavioral results}

For the results described below, unless otherwise specified, $0 \mathrm{~ms}$ is defined as the actual recorded onset of the event of interest in a particular condition; $0^{\circ}$ is defined as the actual correct position the participants were required to report. The clock used in the experiment revolved at a constant speed of $2560 \mathrm{~ms}$ per cycle, which means $1^{\circ}$ equals $\sim 7.1 \mathrm{~ms}$. The transformation from the reported spatial location to time was based on this constant.

The group averages for the reported onset of button press and onset of tone were -35 and $54 \mathrm{~ms}$, respectively $(\mathrm{SD}=47$ and 42 $\mathrm{ms}$, respectively). Both of them differed significantly from $0 \mathrm{~ms}$. The $p$ values were 0.011 and $<0.0005$, respectively (two-tailed one-sample $t$ test). The group averages for the reported position in the action nontiming and auditory nontiming conditions were $0.3^{\circ}$ and $-0.5^{\circ}$, respectively ( $\mathrm{SD}=1.9^{\circ}$ and $2.4^{\circ}$, respectively). Neither of them differed significantly from $0^{\circ}$. The $p$ values were 0.524 and 0.420 , respectively (two-tailed one-sample $t$ test).

The reported onsets of button press were of particular interest and they were assessed individually for every participant where the statistical analyses were performed across trials. Of all 15 participants, 11 of them judged the onset of the button presses to be significantly negative to (earlier than) $0 \mathrm{~ms}$ ( $p<0.05$; onetailed $t$ test). Three participants judged the onset of the button presses to be significantly positive to (later than) $0 \mathrm{~ms}(p<0.05$; one-tailed $t$ test). One participant judged the onset of the button presses to be positive to $0 \mathrm{~ms}$ ( $7 \mathrm{~ms}$ ), but it was not significant ( $p=0.372$; one-tailed $t$ test). These results were further assessed in relation to the fMRI results (see below).

We also analyzed group averages for the latencies of actions, that is, the time of the button press from the onset of a trial. They were 4834 and $4688 \mathrm{~ms}$ respectively ( $\mathrm{SD}=881$ and $646 \mathrm{~ms}$, respectively) for the action nontiming condition and the action timing condition. They did not differ significantly $(p=0.435$; two-tailed $t$ test).

\section{fMRI results}

The following brain areas showed significant changes of activity in relation to the main effect of timing at $p<0.05$ (corrected for multiple comparisons across the entire brain volume): the right frontal eye field, left superior parietal cortex, right intraparietal cortex, and right area MT/V5. All of these areas showed activity of higher intensity in the timing conditions than in the nontiming conditions. These areas are concerned with visual motion (MT/ V5) and visual attention (intraparietal cortex and frontal eye field). Activity is these areas are likely to reflect the need to trace the moving dot as required in this particular task of simultaneity judgment in a visual-spatial context; they do not necessarily re- 
flect the general mechanism for time perception. The details are listed in Table 2 and shown in Figure 1.

There were also brain areas that showed a significant change of activity in relation to the main effect of modality at $p<0.05$ (corrected for multiple comparisons across the entire brain volume). One group included the right primary motor cortex, the supplementary motor area and subjacent cingulate cortex, left supramarginal gyrus and right ventral thalamus: in these areas, there was greater activity in the action conditions than in the auditory conditions. The other area was the right superior temporal sulcus: in this area, there was greater activity in the auditory conditions than in the action conditions. The details are listed in Table 2 and shown in Figure 2.

No brain areas showed any significant change of activity in relation to the interaction of the main effects at $p<0.05$ (corrected for multiple comparisons across the entire brain volume), but because we were particularly interested in testing for timing-related modulations in the actionrelated areas, small volume corrections were performed based on the coordinates obtained in the main effect of modality. This correction is valid because the contrast for the main effect of modality is independent from the contrast for the interaction. Spherical search volumes of a 10 $\mathrm{mm}$ radius were constructed for the four areas that are identified as specific to the action conditions, and the corrections were performed on results assessed at an uncorrected threshold of $p=0.001$. Whereas no significant activation was found in the other three areas, there was a significant interaction of the main effects in the CMA at $p<0.05$ (corrected for multiple comparisons within the search volume). The peak lies on the border between the caudal cingulate motor area and the rostral cingulate motor area. The details are listed in Table 2 and shown in Figure 3.

We then performed a correlation analysis to assess the relationship between this modulation in the CMA and the perceived onset of motor execution. The perceived onsets of the button press was negatively correlated with the enhancement of activity in action timing compared with action nontiming in the CMA ( $r=-0.565 ; p=0.014$; one-tailed). The plot is shown in the bottom left panel in Figure 3. To ensure that the analysis was not dominated by outliers, a conservative nonparametric test of correlation was performed, which undermined the effects of domination by individual subjects (Spearman rank correlation). This correlation was also significant, with a $p$ value even smaller than that obtained from the parametric test $(r=-0.671 ; p=0.003$;

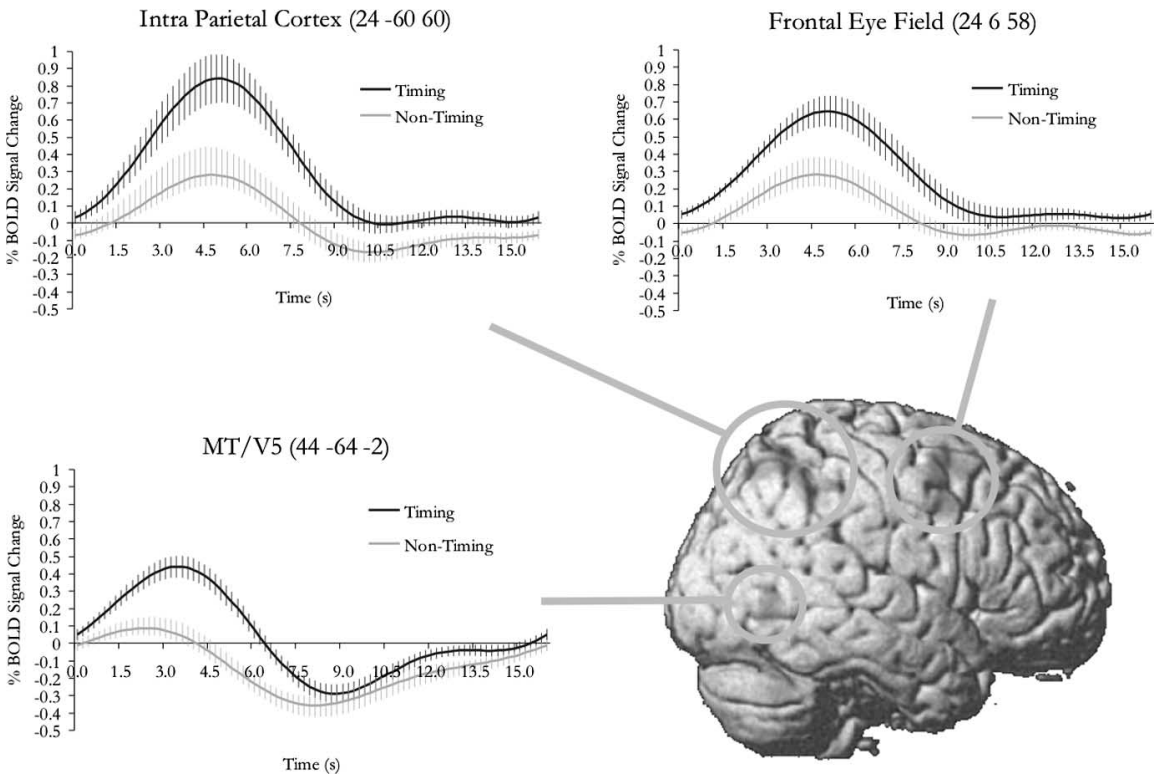

Figure 1. Main effect of timing. Average fitted responses for the time course of the hemodynamic activity were plotted for each area significantly modulated by the main effect of timing. Activity in these areas is likely to reflect the need to trace the moving dot as required in this particular task of simultaneity judgment in a visual-spatial context; they do not necessarily reflect the general mechanism for time perception. The error bars represent SE across subjects.

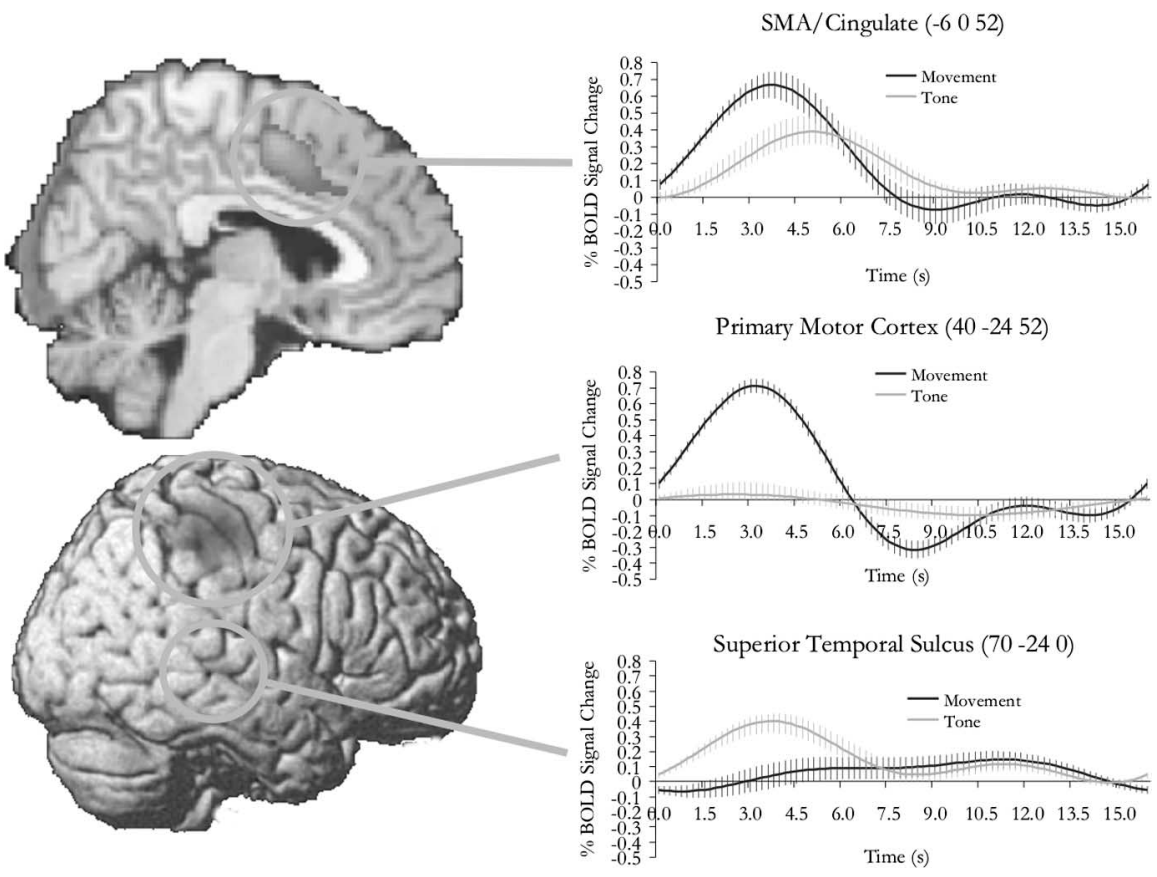

Figure 2. Main effect of modality. Average fitted responses for the time course of the hemodynamic activity were plotted for each area significantly modulated by the main effect of modality. The error bars represent SE across subjects.

one-tailed). This means that the correlation was not driven by outliers.

The correlation analyses were based on the magnitude of the timing-related modulation and perceived onsets of action. We also assessed the relationship between the two in terms of the statistical significance at the individual level. For participants that showed a significantly positive modulation in the CMA at $p<$ $0.05,8$ of 10 of them showed a significantly negative bias in terms 


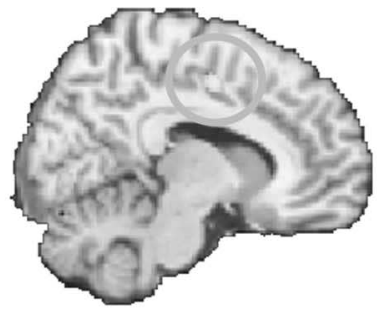

Cingulate Motor Area (CMA; -10-4 48)
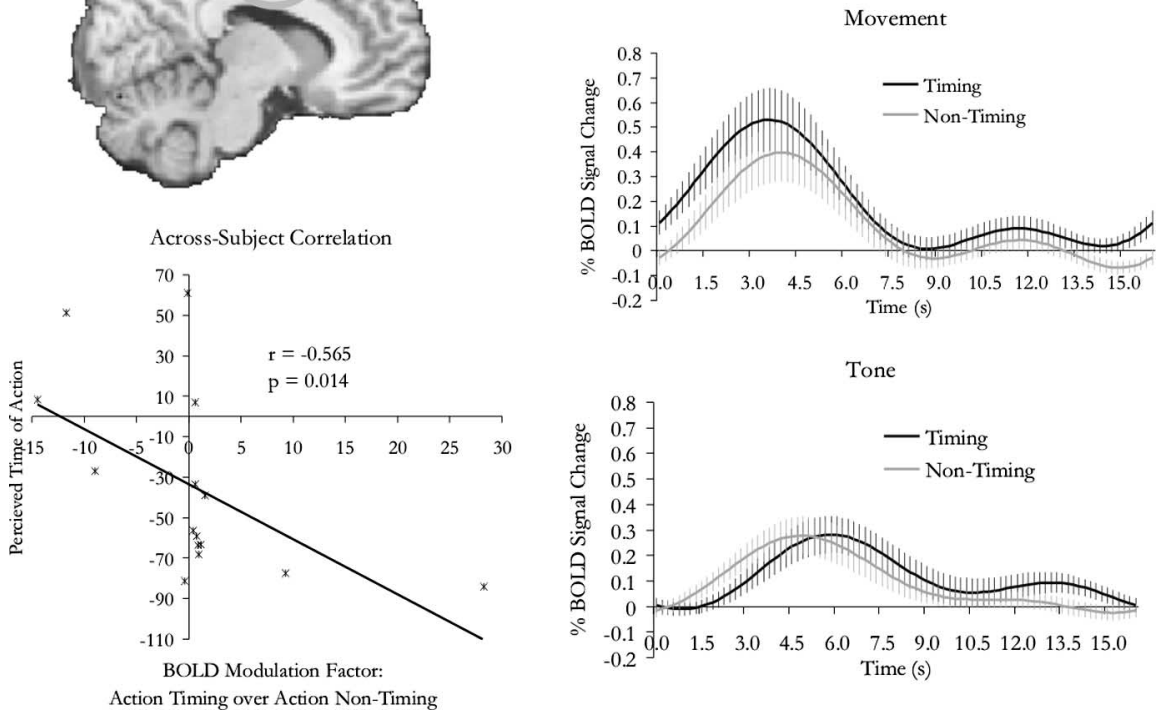

Figure 3. Interaction of timing and modality. Activity in the cingulate motor region was specifically modulated when participants estimated the time of action execution but not when they estimated the onset of a tone. Note that there was also weak activity in the auditory conditions, which was likely to be attributable to general alertness when subjects hear the tone, because activity in these conditions seems to take place at about the time of the onset of the tone; this is inferred with the common assumption that hemodynamic response peaks at $\sim 5-6 \mathrm{~s}$ after stimulus. The activity in the CMA for the action conditions, on the other hand, appears to be attributable to preparation for action, because it peaked substantially earlier. The degree of enhancement specific to action timing (compared with action nontiming) was correlated with the perceived time of action execution. The plot of this correlation is shown in the bottom left panel, where each dot represents an individual participant. Some participants showed an unusually large degree of enhancement of signal, but this correlation was not driven by these outliers, because when a nonparametric test was performed, the correlation only became more significant (supplemental Fig. 1, available at www.jneurosci.org as supplemental material). The error bars represent SE across subjects.
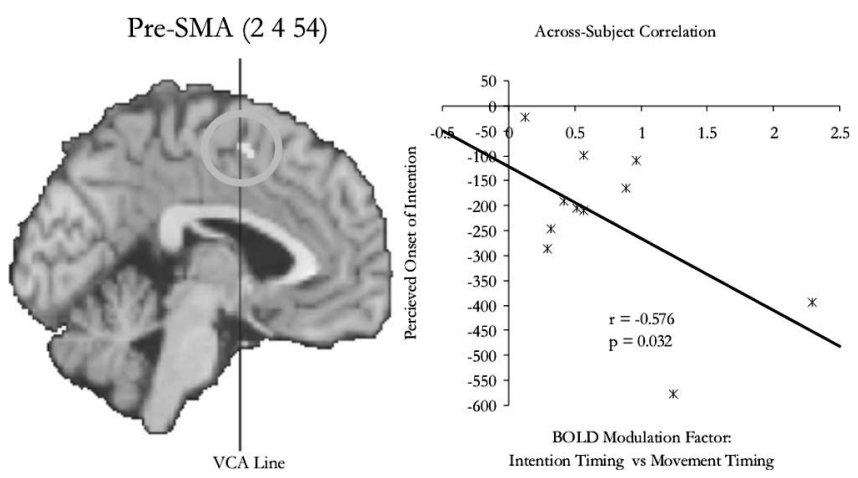

Figure 4. Reanalysis of previous data. A previous study showed that when participants tried to measure and report the onset of intention, compared with the onset of movement, there was enhanced activity in the pre-SMA. Here, we found that the degree of this enhancement was correlated with how early participants thought the onset of intention was. On the scatter plot each dot represents an individual participant.

of their perceived onset of the button press. For participants that showed the modulation at a more standard threshold in imaging analysis $(p<0.001)$, all five of five of them showed a significantly negative bias. Importantly, for the three participants that showed a significantly lack of negative bias at $p<0.05$, none showed any enhancement of activity in the CMA for action timing compared with action nontiming.

\section{Analysis of fMRI data from a previous study on intention}

We were interested in testing whether the relationship between timing-related modulation and the perceived onset of motor execution generalizes to the perceived onset of conscious intentions. Using a similar experiment procedure, we previously conducted an experiment in which the participants estimated either onset of the button press or the onset of their conscious intentions (Lau et al., 2004). Activity in the preSMA was found to be enhanced when participants were required to time their intentions compared with when they were required to time the button presses, and we interpreted the result as reflecting attention to intentions. Based on the correlation results in the current study, we tested whether, across subjects, there was a negative relation between the perceived onset of intention and the degree of enhancement of activity in the pre-SMA. The analysis showed this to be the case ( $r=-0.576 ; p=0.032$; one-tailed). The site of the activation and the correlation plot are shown in Figure 4.

\section{Discussion \\ Estimating the onset of motor execution enhances CMA activity}

The fMRI data suggest that the need to estimate the onset of motor execution is associated with enhanced activity in a CMA. In a previous experiment (Lau et al., 2004), we showed that estimating the onset of intention is associated with an enhancement of activity in the pre-SMA, compared with estimating the onset of action execution. In this experiment, we adopted a more stringent approach and compared the requirement to estimate the onset of action execution (action timing vs action nontiming) with the requirement to estimate the onset of an external stimulus (auditory timing vs auditory nontiming). This ensured that the modulation in the CMA was specific to the need to estimate the onset of action but did not reflect the general process of onset estimation or attention to the visual display, because this is also needed for auditory timing but the level of activity during auditory timing was only similar to that during auditory nontiming (Fig. 3). Importantly, there was no activation found for the main effect of timing in the entire medial frontal region, suggesting this cross-modal timing procedure itself does not induce nonspecific activation in this area.

The CMA projects directly to the spinal cord and the motor cortex (Dum and Strick, 1991; He et al., 1995) and is therefore likely to be related to the processing of information about motor execution. It is possible that estimating the onset of motor execution involves assessing the information represented in this area. We suggest that, in this cross-modal setting, the need to estimate the onset of motor execution is associated with specific enhancement of activity in the relevant motor area, and think this change of activity is best understood as attentional modulation because of the need for deeper processing of the representations associated with action execution. 


\section{Perceptual bias and the onset of motor execution}

The more important finding in this study is that, across participants, the level of the timing-related modulation in the CMA was correlated with the perceptual bias of the timing of the actions: the higher the degree of enhancement of neural activity, the earlier the participants perceived their movements to be. Previous results suggest that we typically judge the time at which we execute actions to be earlier than they actually are (Libet et al., 1983; Haggard and Eimer, 1999; Haggard et al., 1999; Lau et al., 2004; Sirigu et al., 2004); the behavioral results in this experiment also confirm this. The fact that higher degree of enhancement of neural activity was correlated with earlier perception means that this enhancement was associated with perceived onsets that were further away from the actual onset. Analysis of the individual statistical significance of neural modulation and perception bias also suggests that the degree of neural enhancement was particularly correlated with early perception that was significantly erroneous. Additionally, the participants who showed a significant lack of bias did not show the neural modulation effect at all. Because this neural enhancement was consequence of being required to engage in the timing method of Libet et al. (1983), this suggests that the timing method may be problematic in that it contributes to a negative bias in the perceived onset of movements.

The mechanism for this effect could be that the participants are using the increasing neural activity just before the movement to time the movement, and that the task-demand enhances this activity in the CMA areas. This would cause the signal to reach a fixed threshold at an earlier time.

\section{Perception bias and the onset of intention}

The reanalysis of our previous data (Lau et al., 2004) suggests that this pattern of correlation may also operate in the case of intention. In the pre-SMA, there was a correlation between the degree of neural enhancement and the reported onset of intention. We think the mechanism is likely to be similar to the case of the estimation of the onset of movements, in a way that the task demand leads to enhancement of the neural activity, and thus making the signal reach a threshold earlier in time. This is supported by the results of an EEG study by Sirigu et al. (2004), who found that performing the intention task led to earlier onset of the bereistschaftspotential.

The participants that showed the higher enhancement reported the onset to be earlier. From the correlation plot in Figure 4 , one can extrapolate that had there not been any neural modulation in the pre-SMA because of the task demand, and the perceived onset of intention should be only $-120 \mathrm{~ms}$ (i.e., value at $y$-intercept) instead of $-228 \mathrm{~ms}$ as reported in our previous study (Lau et al., 2004).

It could be argued that the early onset of $-228 \mathrm{~ms}$ reflects a more genuine measure of the "earliest possible" time at which intention is felt under the full attention of the participants. Attention is often thought of as enhancing the accuracy or performance of psychological tasks. However, in the context of temporal judgments, attention can lead to biases (Spence et al., 2001). The analysis of the correlation between the neural modulation and perceived onset of movements suggests that the task-induced neural enhancement leads to errors instead of enhanced accuracy. Furthermore, given that we normally do not attend to our intentions as intensely as in performing the tasks in the paradigm of Libet et al., it is unlikely that the $-228 \mathrm{~ms}$ onset reflects the "normal" time at which intention is felt.

The interpretation that the early $-228 \mathrm{~ms}$ onset of intention is exaggerated by the task-demand creates difficulties for models of conscious control of action. Libet et al. (1983) claimed that that, although the onset of intention is later than the onset of the readiness potential, it is earlier than movement execution. Based on this, the authors argued that, although spontaneous actions might be unconsciously initiated, they may nonetheless be abolished by consciousness. Franklin (2000) has formulated this in more specific cognitive terms. The author has reported about a computational model of decision-making based on Baars's global workspace model of consciousness (Baars, 1988). The original demonstration by Libet et al. that intention starts at about -200 ms has been analyzed in relation to the temporal profile of the behavior of the model. The analysis suggests if awareness of intention has functional contribution, one should start to be aware of the intention at a point that is earlier than $-250 \mathrm{~ms}$, to give sufficient time for the conscious editing of intention. However, our data suggest that without the effect of modulation attributable to task-demand, the perceived onset of intention may be as late as $-120 \mathrm{~ms}$. In fact, using the same time estimation paradigm, Sirigu et al. (2004) have reported that patients with lesions in the angular gyrus perceived the onset of intention to be as late as $-50 \mathrm{~ms}$. However, in that report there was no mention that the patients experienced difficulties in controlling their actions consciously, for example in vetoing their actions. Interestingly, it has been suggested that lesion in the angular gyrus can lead to abnormality in time perception (Rorden et al., 1997).

Together, these findings raise the question of whether the conscious control of spontaneous action can be done within a much shorter time window than we had expected, or whether, as suggested by Wegner $(2002,2003)$, our impression of conscious control is simply illusory.

\section{References}

Andersson JL, Hutton C, Ashburner J, Turner R, Friston K (2001) Modeling geometric deformations in EPI time series. NeuroImage 13:903-919.

Baars BJ (1988) A cognitive theory of consciousness. Cambridge, UK: Cambridge UP.

Boring EG (1950) A history of experimental psychology. New York, Appleton-Century-Crofts.

Deecke L (1987) Bereitschaftspotential as an indicator of movement preparation in supplementary motor area and motor cortex. Ciba Found Symp 132:231-250.

Deecke L, Scheid P, Kornhuber HH (1969) Distribution of readiness potential, pre-motion positivity, and motor potential of the human cerebral cortex preceding voluntary finger movements. Exp Brain Res 7:158-168.

Dum RP, Strick PL (1991) The origin of corticospinal projections from the premotor areas in the frontal lobe. J Neurosci 11:667-689.

Franklin S (2000) Deliberation and voluntary action in "conscious" software agents. Neurol Netw World 10:505-521.

Gomes G (1998) The timing of conscious experience: a critical review and reinterpretation of Libet's research. Conscious Cogn 7:559-595.

Haggard P, Eimer M (1999) On the relation between brain potentials and the awareness of voluntary movements. Exp Brain Res 126:128-133.

Haggard P, Newman C, Magno E (1999) On the perceived time of voluntary actions. Br J Psychol 90:291-303.

He SQ, Dum RP, Strick PL (1995) Topographic organization of corticospinal projections from the frontal lobe: motor areas on the medial surface of the hemisphere. J Neurosci 15:3284-3306.

Jezzard P, Balaban RS (1995) Correction for geometric distortion in echo planar images from B0 field variations. Magn Reson Med 34:65-73.

Joordens S, van Duijn M, Spalek TM (2002) When timing the mind one should also mind the timing: biases in the measurement of voluntary actions. Conscious Cogn 11:231-240.

Klein S (2002) Libet's research on the timing of conscious intention to act: a commentary. Conscious Cogn 11:273-279.

Lau HC, Rogers RD, Haggard P, Passingham RE (2004) Attention to intention. Science 303:1208-1210. 
Libet B (1985) Unconscious cerebral initiative and the role of conscious will in voluntary action. Behav Brain Sci 8:529-566.

Libet B, Gleason CA, Wright EW, Pearl DK (1983) Time of conscious intention to act in relation to onset of cerebral activity (readinesspotential). The unconscious initiation of a freely voluntary act. Brain 106:623-642.

Ramnani N, Miall RC (2003) Instructed delay activity in the human prefrontal cortex is modulated by monetary reward expectation. Cereb Cortex 13:318-327.

Rorden C, Mattingley JB, Karnath HO, Driver J (1997) Visual extinction and prior entry: impaired perception of temporal order with intact motion perception after unilateral parietal damage. Neuropsychologia 35:421-433.

Sirigu A, Daprati E, Ciancia S, Giraux P, Nighoghossian N, Posada A, Haggard
P (2004) Altered awareness of voluntary action after damage to the parietal cortex. Nat Neurosci 7:80-84.

Spence C, Shore DI, Klein RM (2001) Multisensory prior entry. J Exp Psychol Gen 130:799-832.

Trevena JA, Miller J (2002) Cortical movement preparation before and after a conscious decision to move. Conscious Cogn 11:162-190.

Wegner DM (2002) The illusion of conscious will. Cambridge, MA: MIT.

Wegner DM (2003) The mind's best trick: how we experience conscious will. Trends Cogn Sci 7:65-69.

Worsley K, Marrett S, Neelin P, Vandal A, Friston K, Evans A (1996) A unified statistical approach for determining significant signals in images of cerebral activation. Hum Brain Mapp 4:58-73.

Worsley KJ, Liao CH, Aston J, Petre V, Duncan GH, Morales F, Evans AC (2002) A general statistical analysis for fMRI data. NeuroImage 15:1-15. 